\title{
Brain Based Learning and Its Relation with Multiple Intelligences
}

\author{
Dr. Ahmad Mohamed Al Ghraibeh \\ College of Education, Department of Psychology \\ King Saud University, PO Box 2458 Riyadh, 1451, KSA \\ E-mail: gha2hmad@yahoo.com
}

Received: December 20, 2011

doi:10.5539/ijps.v4n1p103
Accepted: January 29, $2012 \quad$ Published: March 1, 2012

URL: http://dx.doi.org/10.5539/ijps.v4n1p103

\begin{abstract}
This study aims at exploring the learning that is attributed to the brain and its relationship with multiple intelligences. In order to achieve the goals of the study, two examinations are used. The First one is the examination of the thinking and learning method that is based on both hemispheres of the brain. The second one is the examination of the multiple intelligences. Some referees are consulted for assuring the suitability of the examinations for the measured sample and the calculation of the exam. The sample consists of 300 students who study the course of psychology. The sample is chosen randomly. The results indicates that more repeated method of learning and thinking is based on the left hemisphere of the brain; as it comes out with the highest total of 136 and within a percentage of $(45.3 \%)$. In addition, the results that are related to the dominance of the multiple intelligences indicate that personal intelligence, and physical intelligence are the highest respectively; a mean value of $(49.80 \%)$. Whereas, intrapersonal intelligence comes third with a mean value of (48, 40\%). Finally, musical intelligence scores the lowest mean value.

Regarding connection relation; it is as a statistical function on the level of the $(\alpha=0.05)$ between the natural intelligence and the left hemisphere of the brain on one hand; and the intrapersonal and the integrated intelligence on the other on the other hand.

The study also shows that there is an equal relation with a function at the statistical function of $(\alpha=0.01)$ between the musical intelligence with the right hemisphere and the logical intelligence with the left hemisphere. It is also clear that there is an equal relation between both of (the bodily and the linguistic intelligences) with the left hemisphere and the spatial intelligence with the right hemisphere.
\end{abstract}

Keywords: The multiple intelligences, Learning on brain's hemisphere

\section{Study Background}

Individual distinctions are considered to be the main core of psychology. Many scientists and scholars, who are interested in a profound analysis to the initial phase of the establishment of this knowledge, have discussed it (Jostein, 1999). Recently, some new resources of the individual distinctions have emerged and attracted the attention of the psychologists. These recourses are represented by two recent concepts: Learning styles and multiple intelligences. Actually, those two concepts reflect the multiplicity of cognition that is shaped between individuals and groups in general; and between students in specific. Students differ in their physical, mental and emotional abilities. They also differ in their way of learning, thinking and the kind of intelligence (Klien 2003), (Posner 2004) and (Rees, 2002).

In the past, intelligence was approached as a general peculiarity (I.Q); it is outcome intelligence (I.Q). For example, a person whose intelligence reaches the level of 140 was described as intelligent in everything. Thus, intelligence, based on this way of evaluation, is a general ability. Whereas, the recent vision of intelligence is based on brain research. Although genes still play a role in intelligence, the acquired experience by individuals also plays a role in forming intelligence. In 1983, the definition of intelligence has changed due to what Gardner asserts that each one has multiple intelligences. He defines intelligence as the ability to solve problems (Gardner, 1993). Gardner, in his theory, indicates that each kind of intelligence is separated completely from other kinds of intelligence. Each kind of intelligence has its specific chart for growth; therefore, each individual's mental talents for a specific side do not necessarily approve his ability in another side. For instance, a student's high ability in mathematics does not mean his high level of intelligence in reading, too (Susan \&Karen, 1996). 
After Gardner has identified the evidence that proves having more than one kind of intelligence for Man, he classifies these kinds into nine categories. Gardner's main goal is not defining intelligence but rather classifying it as each person has potential biological abilities. In addition, the difference between individuals is attributed to the kind of intelligence they possessed at their birth; including the method they use to develop it (Gardner, 1983).

During the end of the last century and the beginning of this one, there has been an intensive focus on the resources and the educational system by educators and psychologists. As a result, many concepts have emerged that are related to the topic and gained a great attention. Most of them discuss humans' brain, its curiosity and its thinking; such as: cognition, concentration, imagination, memory, ultra cognition and recognition. Among these concepts, the concept of learning and thinking that reflects the way which a learner uses to process new information, revise and memorize it (Rachford, 2003). Some researchers indicate that this concept is a preference to learning and thinking of an individual (Elliott, Kratochwill, cook \& Travers, 2000); which means that the way for an individual's learning and thinking includes his own focus on a specific part in order to recognize the meaning that is related to the educational subject (Klein, 2003). There are kinds of intelligence work upon a specific partial domain in the brain as it is clearly present in what both Susan and Karen (1996); and Gardner (1993) indicate in identifying the multiple kinds of intelligence in the brain:

Logical-mathematical Intelligence: It is centered in the left half of the brain, the face and the back of both sides of the brain. In these parts of the brain, the human being is able of solving problems and achieving results is the main residence or the basics for science and mathematics. Besides its core function which is to interact with the surrounding things - from ordering to re-ordering things, evaluating its quantity, understanding numeral symbols, understanding quantities and the ability to solve problems with an organized logical way. This kind of intelligence is highly developed amongst engineers, scientists, economists, accountants, detectives and lawyers. Very well-known examples are Albert Einstein and John Dewey.

Linguistic Intelligence: Its main center is the half left hemisphere of the frontal lobe. It is considered the most spread ability among humans as Gardener claims that a person can not continue any effective interaction in the world without recognizing phonetic utterances, structure, semantics, signs or symbols. This kind of intelligence is represented by the ability of writing and the interaction through words. This kind of skills is highly developed among writers, journalists, poets, orators and comedians. Famous examples of people who are distinguished by their linguistics intelligence are Charles Dickens, Abraham Lincoln, T.S. Eliot, and Sir Winston Churchill.

Spatial Intelligence: It is centered in the right hemisphere of the brain. The images that are visualized in the brain are considered useful aids for thinking; hence the visual and spatial imagination is considered a main source for thinking. Therefore, we find this kind of intelligence is represented in the ability of imagination, recognition of the visual world accurately, modifying and switching the initial recognized things by an individual. It also develops individual's visual experience in spite of the absence of the visual material that is related to it. Many of famous scientists have established ideas and concepts out of visual spaces and not by a mathematical thinking. Using this ability allows humans to predict future results. This ability is clearly shown among engineers, architects, artists, sculptors, sailors, photographers, diagram planners and strategic people. Some well-known examples are Picasso and Fran Loud Right.

There is a relationship between the spatial intelligence and the linguistic one which are both equally important. The relationship between the mind and the language is achieved through images and not by voice, and that is because they are both main sources for saving information and resolving problems.

Bodily-kinesthetic Intelligence: this kind of intelligence is centered in the left hemisphere of the brain for the individuals who use right hands and in the right hemisphere of the brain for the individuals who use left hands. This kind of intelligence is shown in the ability of an individual to use his body in a skilful way in order to achieve an expressive aimed goal. This kind of intelligence is represented by accurate movements, dancing, acting and using the skill for solving problems. Surgeons could belong to this category. Some famous examples are Charlie Chaplin and Michael Jordan.

Musical Intelligence: Its main center is the right hemisphere of the brain. This intelligence is considered distinctive from other kinds of intelligences as it is the first emerging intelligence for individuals. It emerges at the age of three. Individuals who have a high level of musical intelligence can compose music and play it by the age of five. While the main functions for this intelligence are in the vocal level, tones and rhythms. This skill is clear among musicians and sound engineers. Most famous examples are Mozart, Leonard Bernstein and Ray Charles.

The students whose musical intelligence is extraordinary and their linguistics intelligence is low use their musical skills to transmit language into rhythmic modes. 
It is noticeable here that this study does not focus on other kinds of intelligence such as naturalistic intelligence, existential intelligence, interpersonal and intrapersonal intelligence as there are not enough literary theories that pinpoint its location in the brain.

\section{Literature Review}

Although there are various studies that discuss the way of learning and thinking with other variables. It seems that studying the way of learning and thinking that is attributed to the brain and its relation to the multiple intelligences is still rare. The study of Roubinek, Bell and Gates (1987) aims at identifying the brain dominance of excellent students. The study uses 184 samples of male and female students from the sixth elementary to the eighth. The results show that best students' answers are related to the right hemisphere of the brain.

While in a study done by Tan (1981), which aims at identifying the way of learning and thinking in a sample of 190 students. The researcher uses Torrens measurement for the way of thinking and learning. The results show that the integral way is dominant among male and female students.

For researching the way of learning and thinking that is attributed to the hemispheres of the brain and its relation with mental talented children's personality, Frances, Steve and Judy (1985) prepare a study that includes a sample of 94 mental talented students whose ages ranged between 9-14 years old. The group is formed and classified depending on the results of testing the way of learning and thinking (the right hemisphere brain's way, the left-hemisphere one, the integral way and the mixture way). The results point that there is a relation between the personal variable factor and favoring a specific technique for treating information.

The study of Junghee and William (1995) aims at recognizing the limitation of favoring the members of the sample to any specific technique of learning and thinking that is based to the brain (right, left, integral) in comparison to their rate of creativity. The sample of the study consists of 193 students of the eleventh level. The study reveals that students with high grades upon the measure of creativity favor the methodology of learning and thinking by using the left hemisphere brain or the integral one.

By considering previous studies in which they navigate the dominant methodology of learning and thinking, it is evident that they differ in their results. The study of Roubinek and Gates (1987) has favored the right hemisphere's learning and thinking way; while Junghee and William's study (1995) has favored the left-hemisphere's. Both of Junghee and William (1995); and Tan (1981) agree on studying the integral way.

Among the studies that explore for the dominance of multiple intelligences, there is a study by Furnham, Callahan and Akanda (2004). The study aims at comparing South Africans and Nigerians in their level of multiple intelligences. The sample consists of 316 university students of white and black ethnicity. They are divided into 181 African and 135 Nigerian students. The researcher uses the measurement of the multiple intelligences. The results indicate that white students are higher in linguistic and bodily intelligence.

In addition, a study of Furnham and Mottabo (2004) aims at identifying the distinctive features in the multiple intelligences especially in a cultural comparative perspective. They consider the cultural difference between students. The comparison is made between Egyptian and British students at university level by testing their IQ base on "Kattel" and the test of their MI. The sample consists of 151 British students and 118 Egyptian students. The results indicate that the Egyptians record higher level in linguistic intelligence along with their higher skills in the following: visual, pronunciation, sensual recognition and accuracy; whereas the British record higher level in mechanical abilities.

Considering the studies that have navigated the dominant kinds of intelligences, it is found that the linguistic and Mathematics (logic) intelligences have recorded higher levels (Furnham, Callahan \& Akanda, 2004). However, in a study by (Furnham \& Mottabo, 2004); it is found that the dominant abilities are the linguistics, visual, pronunciation, fluency, sensual recognition, accuracy and mechanical ones.

Furthermore, to investigate the relationship between the multiplicity of intelligences and the effect of its location in the brain, Yonelinas, et al (2010) have conducted a study to recognize the presented words visually by using the visual implicit memory. They examine the prior claim by testing the visual implicit memory of the patients who suffer from the occipital lobe lesion. The researchers use lexical-decision, mirror-reading, picture-fragment and word-fragment completion tests. They find out that the initial progress for those patients is in its normal level. The results indicate that the right occipital lobe does not necessarily play an important role in visual implicit memory. Also, the left hemisphere can support normal levels of visual priming in a variety of tasks.

The study of Gabriele (1998) aims at evaluating the contribution of the two hemispheres to visual implicit memory. He takes a sample of two patients who suffer from right occipital lobe lesions; which is considered an essential center to the visual implicit memory. He concludes that the right occipital lobe is not critical for visual 
implicit memory. This result is in accordance with the results done by the study of Schacter (1994); and Schacter and Traving (1994).

In a study by Annette (2001), three different music-involvement techniques are compared: Visual representation, verbal description, and control condition. All subjects answer a questionnaire measuring several aspects of responsiveness, such as enjoyment, attention, and understanding. The results indicate that the relation between an activity and the hemisphere of the brain is as the following: In the visual condition, the higher level goes for the dominance of the right hemisphere. However, those with the dominance of the left hemisphere have achieved the higher level of the verbal condition. Also the visual status overlaps with images. The right hemisphere of participants shows a higher ability of drawing visual representations.

A study by Roel, et al (2010) argues that communicative message generation involves perspective taking -mentalizing- which depends on language. The researchers employ a verbal communication paradigm to directly test whether the generation of a communicative action relies on mentalizing. Also, the paradigm is uses to whether the cerebral bases of communicative message generation are distinct from parts of cortex sensitive to linguistic variables. They find that dorsomedial prefrontal cortex, a brain area consistently associated with mentalizing, is sensitive to the communicative intent of utterances.

Regarding the studies that discuss the relation between the two hemispheres of the brain with some kinds of the intelligences, we notice that the study by Yonelinas, et al (2001) confirms the role of the left hemisphere brain in supporting the visual memory. Similarly, the studies of Gabriele (1998), Schacter (1994), Schacter and Tulving (1994) and Squire (1994) deny the existence of the role for the right occipital lobe in supporting the visual memory and the left hemisphere in supporting the verbal ability (Annette, 2001).

\section{Statement of the Problem and Its Questionnaires}

The statement of the problem is defined by revealing the dominant hemisphere of the brain and its relation with the multiple intelligences. This relation adds a significant change to the field of the psychology. In fact most of the current curricula deals only with two kinds of the multiple intelligences; which are the logic/mathematical and the verbal one, even though all multiple intelligences are required for a successful life. With regard to the researcher's knowledge; there are no studies that deal with the dominant hemisphere of brain and its relation with the multiple intelligences. Specifically, the study tries to answer the following questions:

1) What is the level of using the style of learning and thinking that are based on the two hemispheres of the brain in the participants of the sample?

2) What are the dominant kinds of the multiple intelligences for the sample of the study?

3) Taking into consideration that the score between the multiple intelligences and the style of learning and thinking is $(\alpha=0.05)$, so is there any statistical relationship for the level of the dominant hemisphere of the brain for the individuals' sample?

\subsection{Importance of the Study}

From a theoretical aspect, this study adds an important dimension for the educational process, as it sheds light on the relationship between the methodology of learning and thinking that is attributed to the hemispheres of the brain and the multiple intelligences. It also discusses the extent of the accordance between the two concepts; and the accordance between what the students have of intelligences, learning and the way of thinking. Multiple intelligences and the way of learning and thinking contribute to the educational process in an integral way with teaching methodology. It is expected that the results of this study will contribute to solve the problems of the low academic attainment and the low motivation of the learners. When needs and brain-based items are connected to teaching materials, then teaching methods are in harmony with their needs and favored items.

Moreover, the study is theoretically important as is has a special meaning to the two concepts on one side, and the scarcity of such studies that discuss those two concepts on the other side. Actually, this study works in harmony with the researches of the $21^{\text {th }}$ century by focusing on the way in which human's brain learn. It also focuses on the intelligences and methodology that the brain uses to process the information.

\subsection{Related Definitions}

Learning and thinking style: It means the individual's usage of the right or left hemisphere simultaneously in the mental processes. It could be revealed by answering your way of examining.

Right hemisphere for learning and thinking style: the achieved degree by the student due to the application of the (YSOLAT) measure which could be obtained according the correction measurement that is recommended in the examination guidebook and it must be higher than 120 degree. 
Left hemisphere for learning and thinking style: the achieved degree by the student due to the application of the (YSOLAT) measure which could be obtained according the correction measurement that is recommended in the examination guidebook and it must be higher than 120 degree.

Integrated hemisphere for learning and thinking style: the achieved degree by the student due to the application of the (YSOLAT) measure which could be obtained according the correction measurement that is recommended in the examination guidebook and it must be higher than 120 degree.

Multiplicity of intelligences: the achieved degree of the student due to the application of the multiple intelligence measurement which is attributed to Gardener theory that consists of nine kinds of intelligences.

Study Variables: The study includes the following variables: learning and thinking style that is attributed to the two hemispheres of the brain (right, left and integral). Multiple intelligences (logic "Mathematics", linguistic intelligence, spatial intelligence, bodily- kinesthetic intelligence and musical intelligence).

Study limitations: This study is limited to the students of the education department at King Saud University. It is also limited to the study of the psychometric characteristics for examining the way of learning and thinking that is attributed to the brain theory. Then, the achieved results of the current study will be valid to be generalized on the statistical community for this study and for similar statistical communities.

Study Sample: The sample consists of 300 students who study a course of psychology in the faculty of education at king Saud University and it is selected randomly.

Study Tools: the following two tools are used in this study:

First, The learning and thinking style measurement: Which consists of the special copy for adults and which is described as an examination of 36 paragraphs, each includes three statements (options) that reflect the way of student's thinking, and each measures the left hemisphere of the brain. The tester uses one of the required statements (right hemisphere, left hemisphere and the integrated). The tested chooses one statement of each paragraph until he achieves 36 degrees at the end of the examination which is divided into three parts: the right hemisphere, the left hemisphere and the integrated.

Authenticity of the study: Authenticity of the contained information for the measurement of the learning and thinking style is confirmed by research findings as both the right and the left hemisphere apply different operations from each other. The study clarifies that the right hemisphere of the brain is responsible for a higher degree for the following abilities: creativity, art and spatial recognition. In addition to the non verbal abilities that are based on the visual side, the initiative, and construction, the study retrieves the non verbal information and ordering things to find the relation between them. It is also evident that the left hemisphere of the brain is responsible in a clearer way for the verbal abilities like using the language in expression, analysis, logical arrangement in speech, comprehension and production of the language and the retrieving to the verbal information. Due to this, it is clear that the tasks that are included in each paragraph of the test are in accordance with both what previous studies confirm and the specialty of each hemisphere of the brain.

Eight referees, who are faculty members, review and approve the test. The researcher takes their comments into consideration when revising the test; and they all state that the test is suitable for the purpose of the study.

Stability of the measurement: To assure the stability of the examination, the researcher applies it on three sections of university students taking a course in educational psychology from the community of the study and outside the sample. The fixed sample consists of 90 students. The test is applied and reapplied within break duration of two weeks. Then, the researcher calculates the fixed factor by using the fixed exam of Pearson. The total value for the fixed factor is 81.0.

Correction of the measurement: Each test is given a grade for each of the methods of learning and thinking: One for the left brain, the right brain and the integrated. The researchers views participants' favored or dominant style in dealing with different tasks using the measure of the modified standardized degree of 120 or more (its medium is 100 and its standard deviation is 20).

This measurement is built on two bases. First, it is built on what has been achieved so far of analyzing the functions of the brain. Second, it is also built on what researches have achieved in neural and surgical results through the existence of the two main hemispheres that are specialized in their functions. Applying the measurement, each individual gets 3 grades for each methodology of learning and thinking ways (right, left, integrated). It also reveals the favored or the dominant way for each individual in handling different tasks based on a specific criterion- that is ( the justified measurement degree 120 or more, the medium level is 100 and the rotation for it is 20) (Torrance, et al, 1997). 
Second: Multiple intelligences measurement: It is a measurement for multiple intelligences which tests nine kinds of intelligences based on Gardner theory (McKenzie, 1999).

The validity of the measurement: To assure of the logical validity of the measurement; it is reviewed by ten referees of the specialists in psychology. The researcher asks each referee to review the paragraphs, the suitability of the kind of the intelligence that is measured, its clear level, and its suitable structural form. The researcher takes referees' notes into consideration. Then they indicate that the measurement is suitable for the study.

Correction of the measurement: Each kind of multiple intelligences consist of (10) paragraphs that measure the performance of the students on each level of the multiple intelligences. The answer is determined by the paragraph in which a student might feel as suitable to his intelligence ability with a degree of (1); while a degree of ( 0 ) is given to the paragraph that he does not feel that it goes well with his intelligence level. After that, the selected paragraphs are to be gathered and the result is to be multiplied by (10) so the max degree would be out of (100) in each section. The degrees of the students in each section are restricted within the range (10-100), so the degrees between (10-40) are considered a low ability intelligence of an individual, (50-60) is a medium one and (70-100) is a high level of intelligence to an individual (McKenzie, 1999).

The statistical analysis: the numbers and the percentages are calculated for each way of learning and thinking; as the mean value is done on (Pearson Correlation) calculation and the standard deviation to reveal the kinds of multiple intelligences and some equations are inducted to find out the relationship between the way of learning and thinking and the multiple intelligences.

\section{Results of the Study and Discussion}

For answering the first study question: "What is the level of using the learning and thinking ways that are based on the two hemispheres of the brain for the individuals of the sample study?" The recurrences are being calculated along with the percentages of the individuals of this sample within each level of the learning and thinking ways that are attributed to the two hemispheres of the brain. And this is shown in table 1 below:

\section{Insert Table 1 Here}

Table 1 shows that the higher level of recurrences is 136 in the favor of the left hemisphere side with a percentage of recurrence to $(45.3 \%)$.

The surpass of the left hemisphere could be because students deal with a lot of study skills where language is located. Therefore, it is expected that the activity of the hemispheres of the brain depends on the nature of the tasks and activities that are treated by an individual (Belger, 2002) and (Nishiizawa 1994).

The results of this study are compatible with the results of the study of Junghee and William (1995) concerning the dominance of the way of learning and thinking of the left hemisphere. However, the present study differs from the study of Roubinek and Gates (1987) that stands in favor of the way of learning and thinking of the right hemisphere of the brain. Lately, studies of Tan (1981); and Junghee and William (1995) approve the integrated way.

To answer the second question of the study: "What are the kinds of the multiple intelligences of the individuals in this sample?" The researcher calculates the means and standard deviations for the dimensions of multiple intelligences as shown in the table 2 :

\section{Insert Table 2 Here}

It is noticeable from table 2 that the mean values of the measurements of the multiple intelligence dimensions range between $(36.60-54.67 \%)$. Also, the highest value is the Interpersonal (personal). In addition, the bodily intelligence (physical) comes within a mean value of $(49.80 \%)$. At the third rank, there is the intrapersonal intelligence (social) with a mean value of $(48.40 \%)$. It is clear that the main total of the mean value is $(40.61 \%)$.

It is clear that university students are within a high level of the interpersonal intelligence "personal", and that could be due to the circumstances that are established socially in the Saudi society. For instance, the family factor that motivates an individual to organize himself by understanding and realizing his abilities and feelings towards himself and the others; also by proctoring his performance, behavior and personality in order to enjoy a mental concentration and to adopt the dominant social system in a real way. In addition to that, the bodily intelligence "physical" is on the second rank among students and this explains that students prefer the physical way of learning which has occupied the first rank as a preferable way for learning. The fact that the Saudi culture does not go in harmony with music, indicates the low level of the musical intelligence among sampled students.

The results of this study differ from the results of previous results. The researcher finds that the linguistic and 
mathematical seem to be within the highest levels (Furnham, et al, 2004). While a study by Furnham and Mottabo (2004) affirms the dominant ability of verbal, visual, pronunciation, fluency of words, speed of sensual recognition, accuracy and mechanical abilities.

Regarding the third question: "Taking into consideration that the score between the multiple intelligences and the style of learning and thinking is $(\alpha=0.05)$, so is there any statistical relationship for the level of the dominant hemisphere of the brain for individuals' sample?"

The researcher concludes Pearson correlations between the dominant ways of learning and thinking and the kinds of the multiple intelligences. This is shown in the table 3:

Table 3 shows the following:

1) There is a direct correlation with statistical function at the level $(\alpha=0.05)$ between natural intelligence "related to naturalistic" and the left hemisphere. Moreover, that correlation exists between the intrapersonal intelligence "social" and the integrated one. In addition, the correlation is noticeable between the total IQ as a whole and the way of the right hemisphere. The value of the equations is positive and that is statistically shown on at the function level $(\alpha=0.05)$.

\section{Insert Table 3 Here}

2) There is an inverse relation that has a statistical function at the level $(\alpha=0.05)$ between the musical intelligence and the left hemisphere way, the logical intelligence "mathematical" and the right hemisphere way. The inverse relation is also shown between the kinetic intelligence "physical" and the right hemisphere; and between the spatial intelligence "visual" and the left hemisphere way. The value of the results is negative and the statistical function at the level of the function $(\alpha=0.05)$.

3) There is a direct correlation with the function at the statistical function of the level $(\alpha=0.01)$ between the musical intelligence and the right way; and the logical intelligence "mathematical" with the left hemisphere way. It also shows direct correlations between three variables. The first correlation appears between the (kinetic intelligence "physical", linguistic intelligence "verbal") and the left hemisphere. Secondly, the spatial intelligence "visual" and the right way are clearly correlated. Finally, a correlation is noticeable between the IQ and the left hemisphere. The value is positive with a statistical function at the level $(\alpha=0.01)$.

4) There is an inverse relation at statistical function of the level $(\alpha=0.01)$ between all of (the musical intelligence, mathematical "logical" intelligence, kinetic intelligence "physical", linguistic intelligence "verbal", spatial intelligence "visual") and the integrated way, as an inverse relation appeared between the mathematical intelligence "logical" and the right hemisphere way and between the IQ and the integrated way; as the value of the function was positive and a statistical function at the level of the function $(\alpha=0.01)$.

In fact, the left hemisphere of the brain includes the operations of the logical and linguistic analysis. However; the right half of the brain includes operations of the non verbal thinking as processing of the operations that is related to the spatial memory (Sift, 1990). Each half of the brain seems to be able to deal with several kinds of skills but they differ in the range of efficiency and proficiency. Speech and verbal abilities depend on the left hemisphere of the brain. Several skills require the recognition of the surrounding spatial of an individual such as drawings and images. The unclear angles depend on the right half of the brain (Sift 1990) and (Annet, 1985). Concerning spatial ability, the right hemisphere of the brain is more active and it could make a progress to the visual left domain. Thus, we cannot say that both of the halves are separated completely in their performance but the individuals use each half according to the system that is special to spatial abilities (Roig \& Cicero, 1994).

According to Van and Schkade (1989), there is a difference between the two hemispheres. They indicate that the right hemisphere includes specifically the non verbal abilities that depend on the visual, spatial, and sensual recognition; the double recognition of things; the initiative motive, understanding the utterances and musical abilities. Whereas, the left hemisphere of the brain includes verbal abilities, logical utterances and the abilities of a digital, expressive, logical and dialectical analysis.

The individuals who depend more on the right hemisphere of the brain have a higher ability of the retrieval for the nonverbal information (images). On the contrary, the individuals who depend on the left hemisphere of the brain have higher abilities to retrieve the verbal information as what Toombs (1981) indicates. Kinsbourne (1982) believes that the relationship between logical mental operations like ordering things and understanding; and the spatial memory depends on the right hemisphere

As for the relationship between the hemisphere and the motion, both of Tan and Akgun (1992) find out that the left half of the hemisphere of the brain is more attributed to the great motion and that explains the connection of 
the bodily intelligence (physical) with the left half as if all of the individuals in the sample are from the users of the right hand; and they use the bigger movements.

The results of this study agree with the study of Annette (2010) that confirms the role of the right hemisphere in supporting the visual memory, and the left hemisphere of the brain in supporting the verbal ability. However, it differs from the results of a study by Yonelinas, et al (2001) that assures the role of the left hemisphere of the brain in supporting the visual memory. Various studies by Gabrieli, (1998), Schacter (1994) Schacter and Tulving (1994) and Squire (1994) indicate that the nonexistence role of the right occupant lobe in supporting the level of the visual memory.

As it is clearly shown in the results of the study, the researcher finds that musical, linguistic, logical, bodily, and spatial intelligences show a function on the integrated way. Accordingly, there is no extreme dominance for any half of the brain in favor of the other as each half plays a role in each behavior (Schold, 1998) and (Hobler, 1992). It seems that the relationship between of each one of the hemispheres and behavior is too difficult to be mostly explained. It is also hard to recognize the reasons of the relationship (Annett, 1999). The researcher can infer that the dominance of the hemispheres does not go along with the law of wholeness or nothingness. However; does that mean that there is no dominance for any of the two hemispheres of the brain? (Starron \& Manniy, 2005).

Many researchers clarify that individuals tend to depend on using the brain in a wholeness way more than using it partially. For example, the left hemisphere analyzes the verbal language in musical analysis, whereas its appreciation is located on the right hemisphere (Send, 1991) and (Springer \& Dentsch, 1989).

Cohen indicates that the relations are classified according to their strength as the following: Small Effect Size, $r$ $=0.1-0.23$; Medium, $r=0.24-0.36$; Large, $r=0.37$ or Larger. The researcher concludes that the relations are weak or medium even with a statistical function. (The natural intelligence and the left hemisphere way), (the interpersonal intelligence and the right hemisphere; and the integrated ways) (Cohen, 1992).

\section{Recommendations}

The study recommends the following:

1) The necessity for studies that discuss multiple intelligence kinds in general as they are unavailable. Moreover, the researcher recommends other researchers to focus on the following intelligences: (natural, existential, interpersonal and intrapersonal intelligences) because there are not any studies that confirm the location of these intelligences in the brain.

2) Furthering research on the methodology of the learning that is attributed to both hemispheres of the brain and the multiple kinds of the intelligence at different age levels.

\section{References}

Annett, M. (1985). Left, Right, Hand Brain: The Right Shift Theory. London: Lawerencc Erlbanm Associates Ltd.

Annett, M. (1999). Spatial Ability in Subgroups of Left and Right Handers. British Journal Psychology, 83(4), 493-515. http://dx.doi.org/10.1111/j.2044-8295.1992.tb02455.x

Annett, M. (2001). Subgroups Handedness and the Probability of Nonright Preference for Foot or Eye and Nonright-Handed Parent. Perceptual and Motor Skills, 93 (3), 911-914. http://dx.doi.org/10.2466/pms.2001.93.3.911

Belger, D. (2002). Music Therapy Sensory Integration and the Autistic Child. USA: Copyright Donna Williams.

Cohen, J. (1992). A power primer. Psychological Bulletin 112, 155-159. http://dx.doi.org/10.1037/0033-2909.112.1.155

Elliott, S., Kratochwill, T., Cook, J., \& Travers, J. (2000). Educational Psychology (3 Ed). New York: Mc Graw-Hill.

Frances, A., Steve, M., \& Judy, J. (1985). The Relationship between Learning Style Preference and Personality Variables: An Exploratory Investigation with Gifted Students. Gifted Child Quarterly, 29 (4), 172-174. http://dx.doi.org/10.1177/001698628502900407

Furnham, A., \& Mottabo, R. (2004). Sex and culture differences in the estimates of genral and multiple intelligences: a study comparing Britsh and Egyption student. Individual Differences Research, 2 (2), 82-95.

Furnham, A., Callahan, I., \& Akande, D. (2004). Self - Estimates of intelligences: a study in two African. The 
Jounal of Psychology, 138(3), 265-285. http://dx.doi.org/10.3200/JRLP.138.3.265-285

Gardner, H. (1983). Frames of Mind: The theory of multiple intelligences. New York: Basic Books.

Gardner, H. (1993). Frames of Mind: The Theory of Multiple Intelligences. Library of Congress Cataloging-in Publication Data: USA.

Holder, M. (1992). Hand Preference Questionnaires: One gets what one asks for. [Online] Available: http: //www. indiana. edu/ primate/92mphil.html (Feb 6, 2009)

Jostein, M. (1999). Educational Psychology as a Foundation in teacher education. What is educational apasychology about, or what it ought to be? [Online] Available: http://www.nla/no/js/newpage11.htm (October 10, 2011)

Junghee, K., William, B. (1995). The Relationship of Creativity Measures to School Achievement and to Preferred Learning and Thinking Style in a Sample of Korean High School Students. Educational and Psychological Measurement, 55(1), 60-74. http://dx.doi.org/10.1177/0013164495055001006

Kinsbunrne, M. (1982). The Ontogeny of Cerebral Dominance. New York: Academy of Sciences.

Klein, P. (2003). Rethinking the multiplicity of cognitive resources and curricular representations: [Online] Available: http://www.tandf.co.uk/journals (June 7, 2011)

Klein, D. (2003). Rethinking the Multiplicity of Cognitive Resources and Curricular Representation: alternatives to learning Style and Multiple Intelligences. [Online] Available: http://www.tandf.co.uk/journals (June 8, 2011)

Mckenzie, W. (1999). Multiple Intelligence Inventory. [Online] Available: http://surfaquarium.com/MI/inventory.htm (March 11, 2010)

Nishiizawa, S. (1994). Cross- Cultural Effects on Hemispheric Specialization Reflected on a Task Requiring Spatial Discrimination of the Thumb by Japanese and American Students. Perceptual and Motor Skills, 78, 771-776.

Posner, M. (2004). Neural System and Individual Differences. Teachers College Record, 106 (1), 24-30. http://dx.doi.org/10.1111/j.1467-9620.2004.00314.x

Reese, S. (2002). Understanding our Differences. EBSCO (July 17, 2011)

Rochford, R. (2003). Assessing learning style to improve the quality of performance of community writing programs: a pilot study. [Online] Available: http://www.qcc.cuny.edu/is/hournals (June 5, 2011)

Roel, M., Miriam, B., Jan, P., Matthijs, N., Peter, H., \& Ivan, T. (2010). A Dissociation Between Linguistic and Communicative Abilities in the Human Brain. Psychological Science, 21(1), 8-14. http://dx.doi.org/10.1177/0956797609355563

Roig, M., \& Cicero, F. (1994). Hemisphericity style, sex, and performance on a line Bisection task: An exploratory study. Perceptual and Motor Skills, 78, 115-120. http://dx.doi.org/10.2466/pms.1994.78.1.115

Roubinek, L., Bellm, L., \& Gates, A. (1987). Brain hemispheric Perference of intellectually gifted children. Roeper Review, 10, 120-122. http://dx.doi.org/10.1080/02783198709553100

Schacter \& E. Tulving (Eds.) (1994). Memory systems 1994 (pp.233-268). Cambridge, MA: MIT Press.

Schacter, D. (1994). Priming and multiple memory systems: Perceptual mechanisms of implicit memory. In D.L.

Schold, C. (1998). Handedness and Cerebral Dominance. [Online] Available: http://neurology.swmed.edu/ pearls/ pearl3/ pearl3. htm (March 2, 2010)

Send, D. (1991). Human Motor Development. New York .Mayfield Publishing Company .

Sifft, J. (1991). Educational Kinesiology Empowering Students and Athletes through Movement. Physical Education, 5, 28-31.

Springer, S. P., \& Deutsch, G. (1989). Left Brain Right Brain. New York: freeman.

Springer, S. P., \& deutsch, G. (1989). Left brain right brain. New York: freeman.

Strarron, J., \& Manniy. (2005). Mind and Hand: The Birth of MIT, MIT Press .

Susan, J., \& Karen, D. (1996). Exceeding Expectations: A User's Guide to Implementing Brain Research in the Classroom.

Tan, U., \& Akgun, A. (1992). Contributions of the Right and Left Brain to Manual Asymmetry in Hand Skill in Right Handed Normal Subjects International. Journal of Neuroscience. 65 (1-4), 11-17. 
Tan, W. (1981). Cerebral Hemispheric specialization of academically Gifted and female adolescent. The journal of creative behavior, 15 (4), 276-277. http://dx.doi.org/10.1002/j.2162-6057.1981.tb00310.x

Toombs, N. J. (1981). Differences in Lateral eye movement and hemispheric dominance associated with abilility to recall verball and non-verbal stimuli. Dissertation Abstracts International, 42(9).

Torrance, P., Reynolds, R., Ball, F., \& Riegel, R. (1977). Your Style of Learning and Thinking Preliminart norms abbrevialed technical notes, scoring keys, and selected references. Gifted Child Quarterly, 21, 563-573.

Van, D., \& Schkade, I. (1989). The Brain Lemisphere Preferences of Student Teachers with Selected Academic Majors. Teacher Education and Practice, 5 (2), 39-44.

Yonelinas, A., Kroll, N., Baynes, K., Dobbins, I., Frederick, R., Knight, R., \& Gazzaniga, M. (2010). Visual Implict Memory in the Left Hemisphere: Evidence From Patients with Callosotomies and Right Occipital Lobe Lesions. Psychological Science, 12(4), 293-298. http://dx.doi.org/10.1111/1467-9280.00354

Table 1. The recurrences and the percentages of the individual's sample based on the ways of thinking and learning

\begin{tabular}{|l|l|l|}
\hline Way & Recurrence & Percentage \\
\hline Left way & 136 & 45.3 \\
\hline Right way & 64 & 21.3 \\
\hline Integrated way & 100 & 33.3 \\
\hline
\end{tabular}

Table 2. Mean value and standard deviations for the measurements of the intelligence dimensions

\begin{tabular}{|l|l|l|l|}
\hline Dimension & Mean Value & $\begin{array}{l}\text { Standard } \\
\text { Deviation }\end{array}$ & Rank \\
\hline Natural intelligence - that is related to naturalistic & 39.67 & 18.67 & 7 \\
\hline Musical intelligence & 36.60 & 18.28 & 9 \\
\hline Mathematical (Logical) intelligence & 44.53 & 19.37 & 6 \\
\hline Existential intelligence & 47.53 & 22.60 & 4 \\
\hline Intrapersonal intelligence/social & 48.40 & 23.54 & 3 \\
\hline bodily intelligence/physical & 49.80 & 19.34 & 2 \\
\hline Linguistics intelligence/verbal & 39.53 & 17.52 & 8 \\
\hline Interpersonal intelligence/ personal & 54.67 & 25.04 & 1 \\
\hline Spatial intelligence/ visual & 45.40 & 17.49 & 5 \\
\hline Total measurements & 40.61 & 10.31 & \\
\hline
\end{tabular}


Table 3. Pearson Correlation between the learning techniques, dominant thinking and multiple intelligence for individuals in the case study

\begin{tabular}{|l|l|l|l|}
\hline Dimension & Left way & Right Way & Integrated \\
\hline $\begin{array}{l}\text { Natural intelligence } \\
\text { related to naturalistic }\end{array}$ & $0.11^{*}$ & -0.04 & -0.05 \\
\hline Musical intelligence (Logical) & $-0.12^{*}$ & $0.76^{* *}$ & $-0.37^{* *}$ \\
\hline $\begin{array}{l}\text { Mathematical } \\
\text { intelligence }\end{array}$ & $0.74^{* *}$ & $-0.12^{*}$ & $-0.52^{* *}$ \\
\hline Existential intelligence & -0.04 & 0.01 & 0.01 \\
\hline Intrapersonal intelligence/social & 0.10 & $-0.29^{* *}$ & $0.12^{*}$ \\
\hline bodily intelligence/physical & $0.79^{* *}$ & $-0.13^{*}$ & $-0.57^{* *}$ \\
\hline Linguistics intelligence/verbal & $0.77^{* *}$ & -0.05 & $-0.58^{* *}$ \\
\hline $\begin{array}{l}\text { Interpersonal intelligence/ } \\
\text { personal }\end{array}$ & 0.02 & -0.08 & 0.06 \\
\hline Spatial intelligence/ visual & $-0.14^{*}$ & $0.72^{* *}$ & $-0.32^{* *}$ \\
\hline Total measurements & $0.41^{* *}$ & $0.11^{*}$ & $* *-0.38$ \\
\hline
\end{tabular}

*statistical function of the level $(\alpha=0.05)$. ** statistical function of the level $(\alpha=0.05)$ 